\title{
Comparison of pre and post Global longitudinal Strain imaging in thrombolytic and primary percutaneous coronary intervention in acute ST elevation Anterior Myocardial infarction
}

\author{
MOHAMMAD RAYHAN MASUM MANDAL, SYED ALI AHSAN, HARISUL HOQUE, MOHAMMAD FAISAL IBN \\ KABIR, KHURSHED AHMED, FAKRUL ISLAM KHALED, SM EAR-E-MAHBUB, NILUFAR FATEMA. \\ Department of Cardiology, Bangabandhu Sheikh Mujib Medical University, Shahabag, Dhaka. \\ Address of Correspondence: Dr. Mohammad Rayhan Masum Mandal, Assistant professor, Department of Cardiology, \\ BSMMU.Email: rayhanmandal@yahoo.com
}

University Heart Journal 2020; 16(1): 11-15

\begin{abstract}
Introduction:
Myocardial hypokinesia is assessed by Echocardiography which is a non-invasive method. ${ }^{1,2}$ This technique, based on two-dimensional visual evaluation of endocardial wall thickening, is subjective. Inter-observer variability is more as it is dependent on operator experience. ${ }^{3,4}$ and may not identify small but clinically significant changes in myocardial function that are below the threshold of visual resolution. ${ }^{5}$ Tissue Doppler imaging provides a more objective assessment of myocardial contractility but is subject to the confounding effects of cardiac translational motion and passive pathological tethering. ${ }^{6}$ These limitations may be overcome by the measurement of local myocardial deformation parameters with strain and strain rate echocardiography. Strain rate is defined as the rate of deformation in response to an applied force and is determined from the spatial gradient of local myocardial tissue velocities between two points which is calculated from the time integral of strain rate and reflects the magnitude of deformation. ${ }^{7,8}$ These parameters are potentially more accurate and specific measures of local myocardial function and may offer an opportunity to improve the detection of regional abnormalities. ${ }^{8,9}$ This study was designed to determine the role of Global longitudinal strain (GLS) in the detection functional ischaemic zone and assessed myocardial contractility following successful thrombolysis and primary percutaneous coronary intervention (PCI) and compare in between two groups.
\end{abstract}

Methods:

Study design: Prospective observation study.

Study period: January 2019 to July 2019

Study place: Department of Cardiology, Bangabandhu Sheikh Mujib Medical University (BSMMU)
Subjects: The study population was 20 patients who were candidate of thrombolysis and 20 patients under went primary PCI after acute ST elevation myocardial infarction setting.

\section{Exclusion criteria:}

1. Old Myocardial infarction,

2. Dilated cardiomyopathy,

3. Moderate to severe valvular heart disease,

4. Atrial fibrillation,

5. Bundle branch block.

Study protocol: A written informed consent was obtained from all participants. Transthoracic Echocardiography (TTE) and 2D global longitudinal strain (GLS) was performed on the day of admission with acute anterior ST elevation MI. Follow up TTE and 2D GLS was done after 6 weeks of thrombolysis and primary PCI. In Echo study, 2D longitudinal strain was done before thrombolysis or PCI and was discharged the day following the interventional procedure. Echocardiographic data acquisition E9 ultrasound scanner (GE Vingmed, Horten, Norway) was used to obtain conventional two-dimensional and colour tissue Doppler images from the standard apical three chamber, four-chamber and two-chamber views in the left lateral decubitus position. Depth and image sector width were set to obtain the highest possible frame rates $120 \mathrm{f} / \mathrm{s}$. Data from three consecutive cardiac cycles in each view. Wall motion was manually tracked to maintain the sample area in the mid-wall position of the segment being interrogated throughout the cardiac cycle. For each myocardial segment, strain rate was calculated from the spatial derivative of myocardial velocity over a defined sample area of $6 \mathrm{~mm}$ within the mid-myocardial layer by using new ROI. Data from three consecutive cardiac cycles 
were averaged to improve the signal-to-noise ratio and used to determine the mean strain rate profile. This was then integrated over time to derive the mean natural longitudinal strain profile using end-diastole, defined by the onset of the peak R-wave of the ECG trace that is Aortic Valve closure point as the reference point. Myocardial tissue velocity curves from the basal septum were used to define end-systole because aortic valve closure induces a clearly identifiable rapid directional change in the basal septal velocity curve coinciding with the peak negative left ventricular $\mathrm{dP} / \mathrm{dt} .{ }^{10}$ By convention, strain rate and strain are expressed as a negative parameter for shortening and a positive parameter for lengthening. Longitudinal directional changes from the apical window in normal myocardium are characterized by systolic shortening and diastolic lengthening and thus, an increase in the magnitude of systolic shortening would be reflected by more negative strain or strain rate values. The following parameters were measured from the mean strain rate and strain profiles: (Figures 1 and 2). Strain rate (rate of deformation) Peak systolic strain rate (SRsys) — greatest shortening during ejection period. Post-systolic strain rate (SRps) — defined as any shortening that occurs during isovolumetric relaxation. Post-systolic strain rate index (SRps/sys) $=$ SRps/SRsys

In addition, visual assessment of wall motion score from grey-scale B-mode cineloops was performed by an expert investigator. Myocardial wall segments were scored according to American Society of Echocardiography recommendations: normal - 1, hypokinetic - 2, akinetic - 3 , and dyskinetic -4 .

\section{Data and statistical analysis:}

The ventricular territory was analyzed by assigning segments in the mid- and basal anterior septum and anterior wall, mid-septum and apex to the left anterior descending artery that adopted by the American Society of Echocardiography. ${ }^{12}$ The allocated segments were considered to be in the culprit territory then the respective coronary artery was the target for intervention in 20 patients (Group A) and thrombolysis in another 20 patients (Group B). Data were analyzed using SPSS for Windows 12.0. Means and standard deviations were used to describe continuous variables. Data were tested for normal distribution. Post-systolic strain rate index and Postsystolic strain index were positively skewed. Within the Group A and Group B groups, paired t-tests were used to compare segments pre-PCI with post-PCI and pre thrombolysis with post thrombolysis. The test results are presented as two-tailed values and statistical significance was inferred at $\mathrm{P} \leq 0.05$.

Results:

A total of 42 acute ST elevation Anterior MI patients consented to participate in the study and they have been offered for primary PCI. 20 patients went for primary PCI who were marked as Group A. 20 patients declined to attend for primary PCI, they were thrombolyed and included in Group B. In addition, two patients had inadequate echo windows at baseline and were excluded. Therefore, the study population was composed of 10 women and 30 men with a mean (SD) age of 44.5 (4.0) years.

\section{Table-I}

Male and female ratio were same in 2 groups

\begin{tabular}{lcc}
\hline & Men (70\%) & Women (30\%) \\
\hline Group A & 16 & 4 \\
Group B & 14 & 6 \\
\hline
\end{tabular}

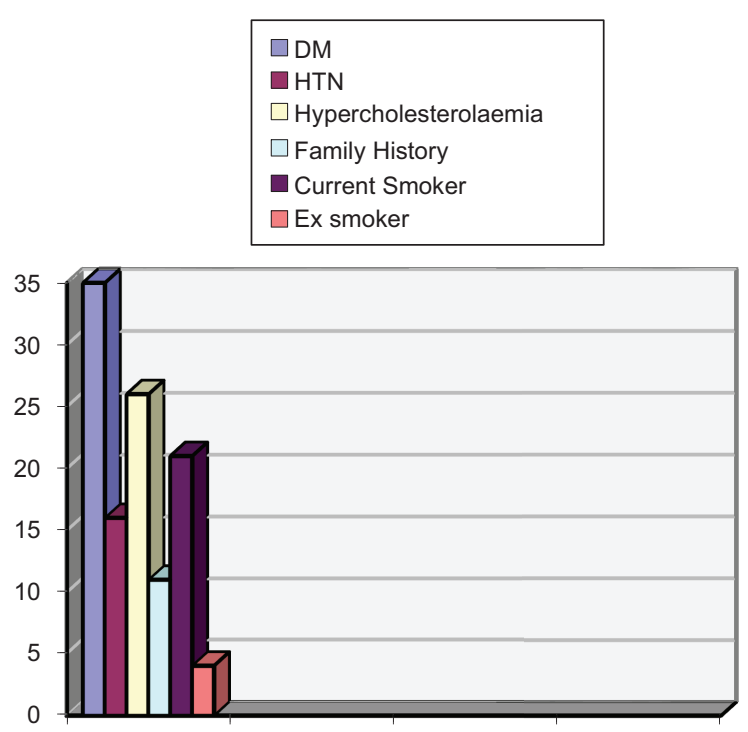

Fig-1: Cardiovascular risk factors

Two had a previous cerebrovascular accident but none had a history of peripheral vascular disease or previous myocardial infarction. Cardiovascular risk factors included diabetes (35), hypertension (16), hyper cholesterolaemia (26), family history (11), current smoker (21), and exsmoker (4).

On admission Echocardiography, hypokinesia were observed in 7 segments of LAD territory ( Mid segment of antero- septal wall, Mid \& apical segments of Septum, mid 
\& apical segments of Lateral wall and mid \& apical segments of anterior wall) and strain rate were obtained which was significantly reduced in LAD territory in both group A and group B. Although a relatively small number of patients was recruited, the study involved data analysis from 7 of myocardial segments (pre- and post-PCI) and was thus adequately powered to detect within patient variation, but not inter-patient variation.

In Group A, the thrombus were found in left anterior descending artery (LAD) ( $\mathrm{n}-15)$ and associated other coronary lesion in right coronary artery (LAD with RCA) (n-2), and left circumflex (LAD with LCX) (n-3) during CAG. Then PTCA was done in culprit coronary lesion in LAD only. PCI was performed using standard techniques and stents were implanted in all patients with good results. At the follow-up visit of 6 weeks time, almost all patients reported a complete resolution of angina. 2D longitudinal strain rate were significantly increased in 17 patients following revascularization in Group A after comparison with pre PCI echo with strain ( $-10 \%$ to $-19 \%)$. Three (3) patients developed significant ST depression and hospitalization was needed. Among them GLS was not increased in 2 patients (-9\% to $-10 \%)$ and mildly increased in 1 patient $(-10 \%$ to $-14 \%)$ in 6 weeks time. The observer made these assessments in a blinded fashion with an interval of 6 weeks. The mean (SD) relative difference in the measurements was $12 \%, 9 \%$, and $8 \%$ at baseline and $17 \%, 13 \%$, and $11 \%$ after 6 weeks for SRsys, Esys, and tSRsys respectively.

Table-II

Mean relative difference in baseline and 6 weeks after PCI in acute MI (Group A)

\begin{tabular}{lccc}
\hline & SR sys & E sys & t-SR sys \\
\hline Base Line (AMI setting) & $12 \%$ & $9 \%$ & $8 \%$ \\
6 weeks after PCI & $17 \%$ & $13 \%$ & $11 \%$ \\
\hline
\end{tabular}

\section{Table-III}

Change of GLS in Group A

\begin{tabular}{lccc}
\hline & $\begin{array}{c}\text { Base Line } \\
\text { (AMI setting) }\end{array}$ & $\begin{array}{c}\text { 6 weeks I } \\
\text { after PC }\end{array}$ & p-value \\
\hline GLS improved & $-10 \%$ & $-19 \%$ & 0.03 \\
significantly (n-17) & & $-14 \%$ & 0.17 \\
GLS mildly improved (n-1) & $-10 \%$ & $-10 \%$ & 1.11 \\
\hline
\end{tabular}

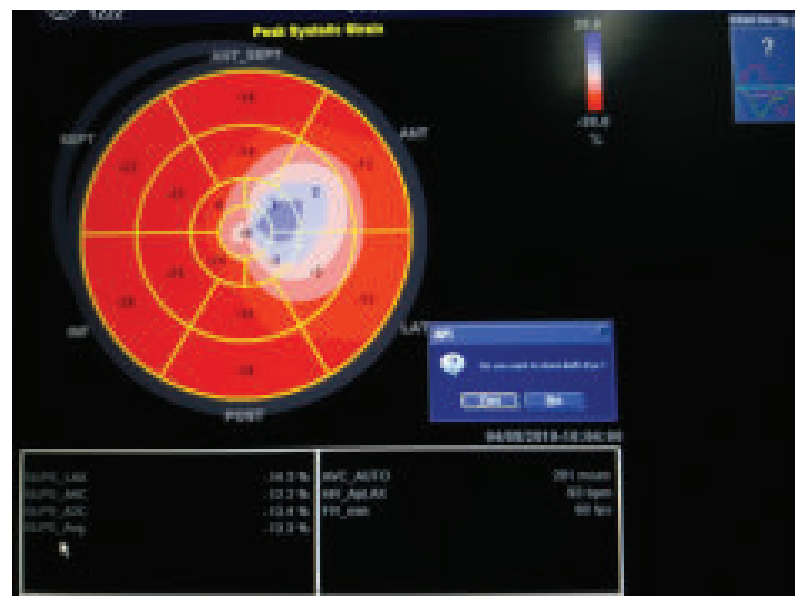

Fig.-2: Bull's eye of GLS in Acute MI 'X'patient.

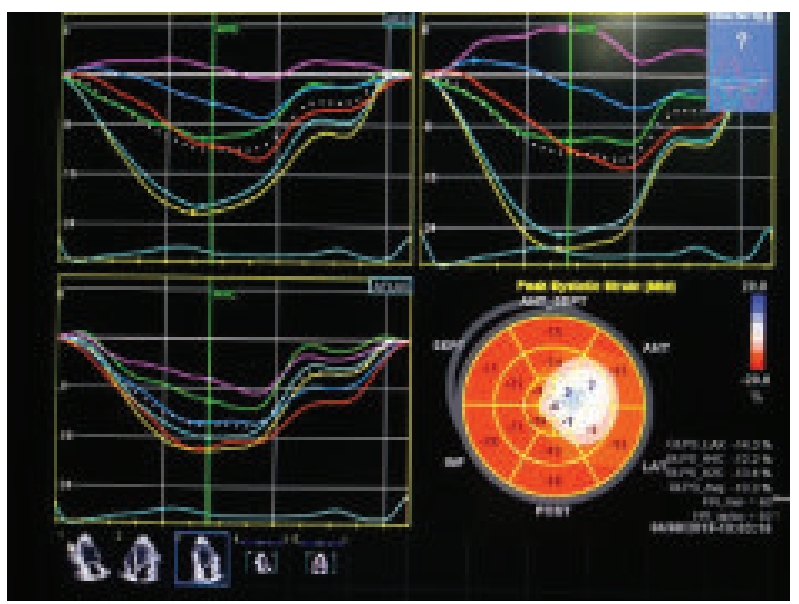

Fig.-3: 2D GLS curves in Acute MI 'X' patient.

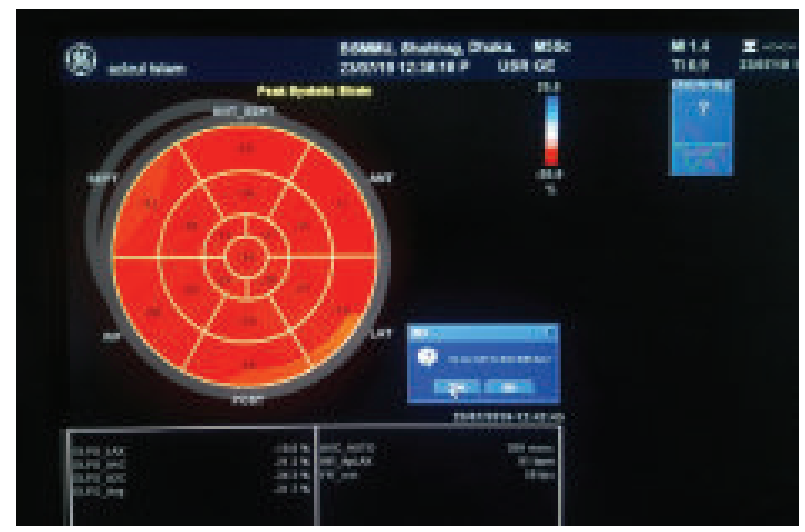

Fig.-4: Bull's eye GLS of 'X' patient 6 weeks after primary PCI

In Group B, thrombolysis was done by streptokinase in 20 patients and hypokinesia was observed in LAD 
territory by ECG and Echo. Then strain rate were obtained which was significantly reduced in same territory. These patients group were discharged with medication and advice for life style modification. In 6 weeks follow up time hypokinesia was observed in same territory in 11 patients and GLS was obtained. GLS was increased ($10 \%$ to $-19 \%$ ) in 9 patients significantly but remained same $(-10 \%$ to $-11 \%)$ in 8 patients as admission and only 3 had mildly improved GLS (-9\% to $-15 \%)$.

\section{Table-IV}

Mean relative difference in baseline and 6 weeks after thrombolysis in acute MI (Group B)

\begin{tabular}{lccc}
\hline & SR sys & E sys & t-SR sys \\
\hline Base Line (AMI setting) & $11 \%$ & $10 \%$ & $9 \%$ \\
6 weeks after thromobolysis & $14 \%$ & $13 \%$ & $12 \%$ \\
\hline
\end{tabular}

Table-V

Change of GLS in Group B

\begin{tabular}{lccc}
\hline & $\begin{array}{c}\text { Base Line } \\
\text { (AMI } \\
\text { setting) }\end{array}$ & $\begin{array}{c}6 \text { weeks } \\
\text { after } \\
\text { thrombolysis }\end{array}$ & p-value \\
\hline $\begin{array}{l}\text { GLS improved } \\
\text { significantly (n-9) }\end{array}$ & $-10 \%$ & $-19 \%$ & 0.08 \\
GLS mildly improved (n-3) & $-9 \%$ & $-15 \%$ & 0.14 \\
GLS unchanged (n-8) & -10 & $-11 \%$ & 1.23 \\
\hline
\end{tabular}

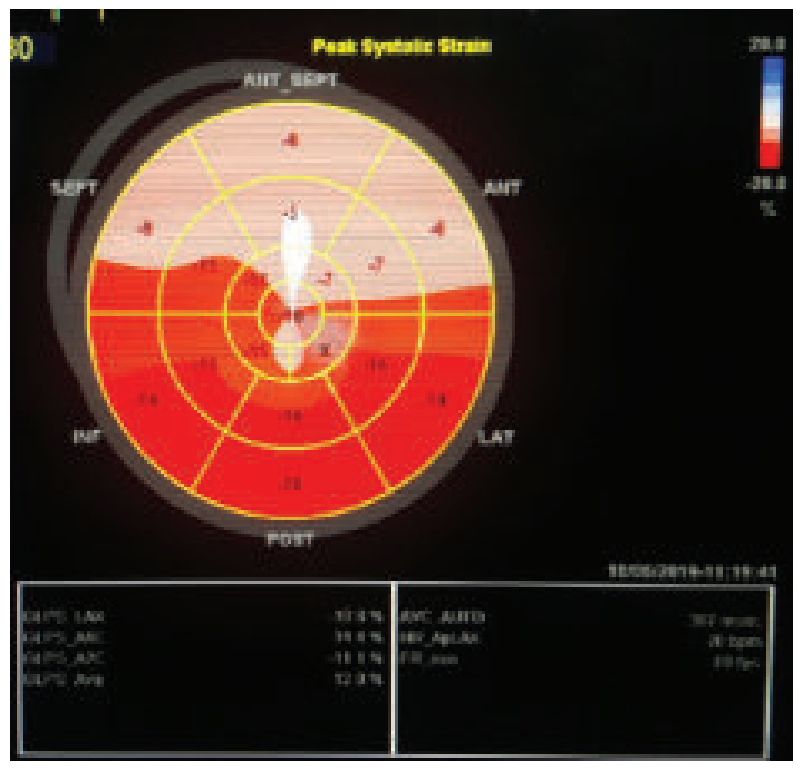

Fig.-5: Base line Bull's eye GLS of ' $Y$ ' patient of STEMI (Anterior, Septal and Apex)

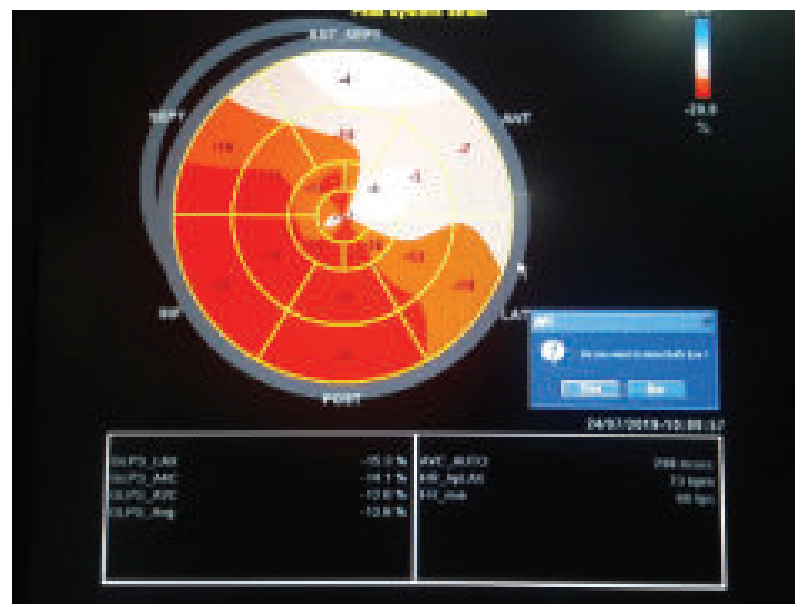

Fig.-6: Bull's eye GLS of ' $Y$ ' patient of STEMI (Anterior, Septal and Apex) in 6 weeks after thrombolysis

These data suggest that SRps and SRps/sys may be able to predict an improvement in myocardial contractility and are potential markers of functional recovery following PCI or after thrombolysis.

\section{Discussion:}

Visual evaluation may erroneously interpret the development of post-systolic contraction as true systolic contractility and fail to recognize that a significant portion of deformation was occurring after aortic valve closure. SRI may overcome these limitations by offering a frame rate sufficient to resolve the timing and distinguish the true peak magnitude of brief local mechanical events. ${ }^{13}$ This is an important finding and raises the possibility of a greater applicability of this imaging modality. In contrast to the present study, patients had significantly lower peak systolic velocities, strain and strain rates on admission than reference value. Persistent changes in recovery were identified in peak systolic strain but post-systolic indices were assessed. The present study did not measure diastolic parameters but the other conflicting findings may be explained by methodological differences. The findings are adjudged all segments supplied by the target artery to be in the 'at risk' territory without considering the relationship between location of the culprit lesion and local myocardial function but these segments may not all be equally susceptible to the effects of ischaemia. Although the estimation of the segmental extent of coronary artery disease is difficult, it may be accomplished using the coronary artery jeopardy score. ${ }^{14}$ Angiography was not performed at follow-up and thus, the presence of subclinical restenosis could not be excluded. However, all but 3 patients reported an improvement in symptoms and there was a significant increase in GLS suggesting that 
ischaemia had been relieved in the territory of the target vessels after PCI in Group A. Whereas in Group B only 8 patients showed significant improvement of GLS after 6 weeks of thrombolysis. Respiratory motion artefact reduced the number of satisfactory traces obtained from the anterior segments. Thus, adequate waveforms were possible in all seven segments but it is important that this is documented so that future studies can concentrate in certain key segments.

\section{Study Limitation:}

Sample size was small. Group B patients were suspected as LAD territory ST elevation MI on basis of ECG and Echo findings but it was not confirmed by coronary angiogram.

\section{Conclusion:}

This study demonstrated a marked reduction of kinesia and in GLS parameters during ST elevation acute Anterior MI on admission. A significant improvement of GLS was observed in primary PCI patients than thrombolysis group after 6 weeks time in acute MI setting.

\section{References:}

1. Ryan T, Vasey CG, Presti CF, O'Donnell JA, Feigenbaum H, Armstrong WF. Exercise echocardiography: detection of coronary disease in patients with normal left ventricular wall motion at rest. J Am Coll Cardiol 1988;11:993-9.

2. Krahwinkel W, Ketterler T, Godke J, Wolfertz J, Ulbricht LJ, Krakau I et al. Dobutamine stress echocardiography. Eur Heart J 1997;18: D9-D15.

3. Hoffmann R, Lethen H, Marwick T, Arnese M, Fioretti P, Pingitore A et al. Analysis of interinstitutional observer agreement in interpretation of dobutamine stress echocardiograms. J Am Coll Cardiol 1996;27:330-6.

4. Picano E, Lattanzi F, Orlandini A, Marini C, L'Abbate A. Stress echocardiography and the human factor: the importance of being expert. J Am Coll Cardiol 1991;17:666-9.

5. Kvitting JP, Wigstrom L, Strotmann JM, Sutherland GR. How accurate is visual assessment of synchronicity in myocardial motion? An In vitro SRI pre- and post-PCI 653 Downloaded from https://academic.oup.com/ehjcimaging/article-abstract/ 9/5/646/2400234 by guest on 17 April 2019 study with computer-simulated regional delay in myocardial motion: clinical implications for rest and stress echocardiography studies. J Am Soc Echocardiogr 1999;12:698-705.

6. Miyatake K, Yamagishi M, Tanaka N, Uematsu M, Yamazaki $\mathrm{N}$, Mine $\mathrm{Y}$ et al. New method for evaluating left ventricular wall motion by color-coded tissue Doppler imaging: in vitro and in vivo studies. J Am Coll Cardiol 1995;25:717-24.

7. Mirsky I, Parmley WW. Assessment of passive elastic stiffness for isolated heart muscle and the intact heart. Circ Res 1973;33:233-43.

8. Heimdal A, Stoylen A, Torp H, Skjaerpe T. Real-time strain rate imaging of the left ventricle by ultrasound. J Am Soc Echocardiogr 1998;11: 1013-9.

9. Abraham TP, Nishimura RA, Holmes DR Jr, Belohlavek M, Seward JB. Strain rate imaging for assessment of regional myocardial function: results from a clinical model of septal ablation. Circulation 2002;105:1403-6.

10. Jamal F, Kukulski T, Strotmann J, Szilard M, D’Hooge J, Bijnens B et al. Quantification of the spectrum of changes in regional myocardial function during acute ischemia in closed chest pigs: an ultrasonic strain rate and strain study. J Am Soc Echocardiogr 2001;14:874-84.

11. Cain P, Marwick TH, Case C, Baglin T, Dart J, Short L et al. Assessment of regional long-axis function during dobutamine echocardiography. Clin Sci (Lond) 2001;100:423-32.

12. Armstrong WF, Pellikka PA, Ryan T, Crouse L, Zoghbi WA. Stress echocardiography: recommendations for performance and interpretation of stress echocardiography. Stress Echocardiography Task Force of the Nomenclature and Standards Committee of the American Society of Echocardiography. J Am Soc Echocardiogr 1998;11:97-104.

13. Kukulski T, Jamal F, D’Hooge J, Bijnens B, De Scheerder I, Sutherland GR. Acute changes in systolic and diastolic events during clinical coronary angioplasty: a comparison of regional velocity, strain rate, and strain measurement. J Am Soc Echocardiogr 2002;15:1-12.

14. Califf RM, Phillips HR 3rd, Hindman MC, Mark DB, Lee KL, Behar VS et al. Prognostic value of a coronary artery jeopardy score. J Am Coll Cardiol 1985;5:1055-63. 